\title{
Meaningful survival benefit for single lung transplantation in idiopathic pulmonary fibrosis patients over 65 years of age
}

To the Editor:

Idiopathic pulmonary fibrosis (IPF) is a chronic lung disease that results in progressive respiratory failure and death. Antifibrotic therapy has been shown to slow disease progression [1,2]. For many patients [3], lung transplantation is the only intervention recognised to provide significant survival benefit [4]. IPF commonly affects older patients, with a median age at diagnosis of 66 years old [5]. Without transplantation, the median survival from diagnosis is 2-4 years [5]. However, registry data indicates impaired outcomes following lung transplantation for older patients [6]. These data have prompted many centres in Europe to avoid offering transplantation to older IPF patients.

IPF patients are particularly negatively impacted when age-specific restrictions (e.g. 65 years or older) to transplantation are employed. In the USA, 33\% of all lung transplants are performed for IPF patients and $\sim 50 \%$ of recipients are aged $>65$ years. By comparison, in the Eurotransplant region, $11.5 \%$ of lung transplants were performed for IPF in 2017 [7]. Of these, only $12.2 \%$ (nine patients) were aged over 65 years [7]. This wide variation in activity highlights how a one size fits all approach can negatively impact on candidates who have the potential to benefit from transplantation.

We compared post-transplant outcomes of IPF patients aged over 65 years of age to those aged under 65 years at the time of transplant. In addition, we performed an intention-to-treat analysis to provide a more informative measure of the utility of lung transplant by incorporating the impact of death on the waiting list [8].

This retrospective study included all IPF patients who were placed on the single lung transplant (SLTx) waiting list at the Irish National Lung Transplant Program between May 2005 and September 2017. Data were captured on 30 September, 2017.

The lung allocation score (LAS) [9] was not utilised for donor allocation but was prospectively calculated to benchmark patient profiles. For optimal physical functionality, all patients were subjected to rehabilitation, and this was monitored and assessed on a weekly basis via phone contact. All patients had a body mass index $(\mathrm{BMI}) \leqslant 28$, as per programme policy

IPF patients were preferentially listed for SLTx. The aim was to maximise donor utilisation and reduce waiting list mortality. Excluded from this approach were IPF patients with severe secondary pulmonary arterial hypertension, who were listed for double lung transplant $(\mathrm{n}=6)$.

Following a donor offer, organ allocation was determined by a real-time multidisciplinary (surgical and medical) evaluation of donor and recipient characteristics. Organ allocation was based on donor/recipient blood group and matching of predicted total lung capacity (TLC). SLTx was offered to IPF patients when there was significant unilateral donor lung pathology. Where there was no donor graft dysfunction, double lung was offered preferentially to cystic fibrosis patients. During the study period 107 double lung transplants were performed.

Kaplan-Meier survival analysis was performed on all IPF patients listed for SLTx between January 2005 to September 2017, comparing those who underwent lung transplant versus those who did not. Survival

@ERSpublications

This study demonstrates a clinically meaningful survival benefit of lung transplantation for IPF patients aged $\geqslant 65$ years http://bit.ly/39pwH2h

Cite this article as: Riddell $\mathrm{P}$, Kleinerova J, Eaton D, et al. Meaningful survival benefit for single lung transplantation in idiopathic pulmonary fibrosis patients over 65 years of age. Eur Respir J 2020; 56: 1902413 [https://doi.org/10.1183/13993003.02413-2019]. 
analysis was performed comparing outcomes of patients aged 65 years or over, versus those under 65 years of age. "Intention-to-treat" analysis was also performed to provide a pragmatic assessment of the treatment effect for all patients who are listed for lung transplant, rather than isolated post-transplant outcomes [10].

Data are expressed as mean \pm SD or number (percentage). Continuous variables were analysed by unpaired t-tests and nominal variables compared using Chi-squared tests. Survival was plotted using Kaplan-Meier curves, with univariate analysis performed using the log-rank score. Hazard ratios and 95\% confidence intervals of these ratios were also calculated to compare survival outcomes. A $p<0.05$ (two-tailed) was considered statistically significant. Analysis was performed using GraphPad Prism 8.0.1 software.

During the study period, 156 IPF patients were listed for lung transplantation in accordance with the International Society of Heart and Lung Transplant guidelines for selection of candidates. 84 patients underwent SLTx for IPF. All cases had usual interstitial pneumonia confirmed on explant histology. 41 patients underwent left SLTx and 43 patients right SLTx. At the time of listing for transplant, the mean LAS of recipients was 43.2 (range 31.7-83.1). Lung function included a mean forced vital capacity, TLC and diffusing capacity of the lung for carbon monoxide of $60.2 \%, 60.7 \%$ and $28.5 \%$, respectively. 19 patients $(22.6 \%)$ had evidence of secondary pulmonary hypertension on right heart catheterisation. Seven patients $(8.3 \%)$ required coronary artery stenting prior to listing. Three had coexisting emphysema.

The mean donor age was 47.0 years (ranging from 17 to 72 years).42.9\% were non-smokers. $78.7 \%$ of donor chest radiographs were normal on the side utilised for transplantation. The mean cold ischaemic time was $313 \pm 153 \mathrm{~min}$. There were no significant differences in baseline characteristics including lung function and LAS comparing IPF patients aged $<65$ years to those $\geqslant 65$ years (table 1 ).

Following transplantation, the mean length of mechanical ventilation was $2.7 \pm 2.7$ days. The mean post-transplant hospital stay was 24.9 days (range 10-230 days). Grade 3 primary graft dysfunction (PGD) occurred in $11.9 \%$ of patients at $72 \mathrm{~h}$. In the 12 months following SLTx, $24.6 \%$ of patients developed one or more episodes of acute rejection (biopsy $\geqslant A 2$ ).

There was no statistical difference between age groups in terms of PGD, days ventilated, length of stay or hospital mortality comparing patients aged $<65$ versus those $\geqslant 65$.

61 IPF patients died on the lung transplant waiting list. The median waiting list time prior to transplantation was 0.37 years. The median time from waiting list activation to death (without transplantation) was 0.68 years. $11 \mathrm{IPF}$ patients were alive and active on the lung transplant waiting list at time of data capture. The Kaplan-Meier survival for patients who did not undergo transplantation was $81.4 \%, 64.2 \%$ and $27.0 \%$ at 3, 6 and 12 months, respectively. The Kaplan-Meier estimated median survival following SLTx was 9.6 years. When comparing the characteristics of patients who died on the waiting list versus those who underwent lung transplantation, there was no significant difference in pulmonary function, 6-min walk test, mean pulmonary artery pressures or LAS at the time of listing for transplant. Both groups had comparable death rates while awaiting transplant. Intention-to-treat analysis indicates a significant benefit for patient undergoing transplant compared to those who do not receive a transplant (figure 1a).

TABLE 1 Recipient characteristics: single lung transplant for idiopathic pulmonary fibrosis (IPF)

\begin{tabular}{|c|c|c|c|c|}
\hline & Overall & IPF patients $<65$ years & IPF patients $\geqslant 65$ years & p-value \\
\hline Subjects $\mathrm{n}$ & 84 & 48 & 36 & \\
\hline Age at transplantation years & $63.3 \pm 0.7(41.1-75.7)$ & $59.1 \pm 0.72(41.1-64.9)$ & $69.0 \pm 0.39(65.1-75.7)$ & $<0.0001$ \\
\hline Males & $83.3 \%$ & $87.5 \%$ & $77.8 \%$ & 0.24 \\
\hline FVC \% pred & $60.2 \pm 1.8(25-96)$ & $58.4 \pm 2.2(25-91)$ & $62.6 \pm 2.9(35.6-96)$ & 0.25 \\
\hline$D_{\text {Lco }} \%$ pred & $28.5 \pm 1.1\left(N^{\#}-46.9\right)$ & $26.9 \pm 1.5\left(N A^{\#}-45.6\right)$ & $30.7 \pm 1.7\left(\mathrm{NA}^{\#}-46.9\right)$ & 0.10 \\
\hline 6MWT m & $230.8 \pm 14.8(10-495)$ & $210.5 \pm 18.9(10-405)$ & $255.9 \pm 23.5(30-495)$ & 0.13 \\
\hline Lung allocation score & $43.2 \pm 1.3(31.7-83.1)$ & $44.8 \pm 1.8(31.7-83.1)$ & $41.1 \pm 1.6(33.3-72.9)$ & 0.14 \\
\hline
\end{tabular}

Data are presented as mean \pm SD (range), unless otherwise stated. BMI: body mass index; FVC: forced vital capacity; $D_{\text {Lco: }}$ carbon monoxide diffusing capacity; PA: pulmonary artery; 6MWT: 6-min walk test. ${ }^{\#}$ : $D_{\text {Lco }}$ was unable to be recorded for 16 of 84 patients (nine with age $<65$ and seven aged $\geqslant 65$ years), due to low lung volumes. 

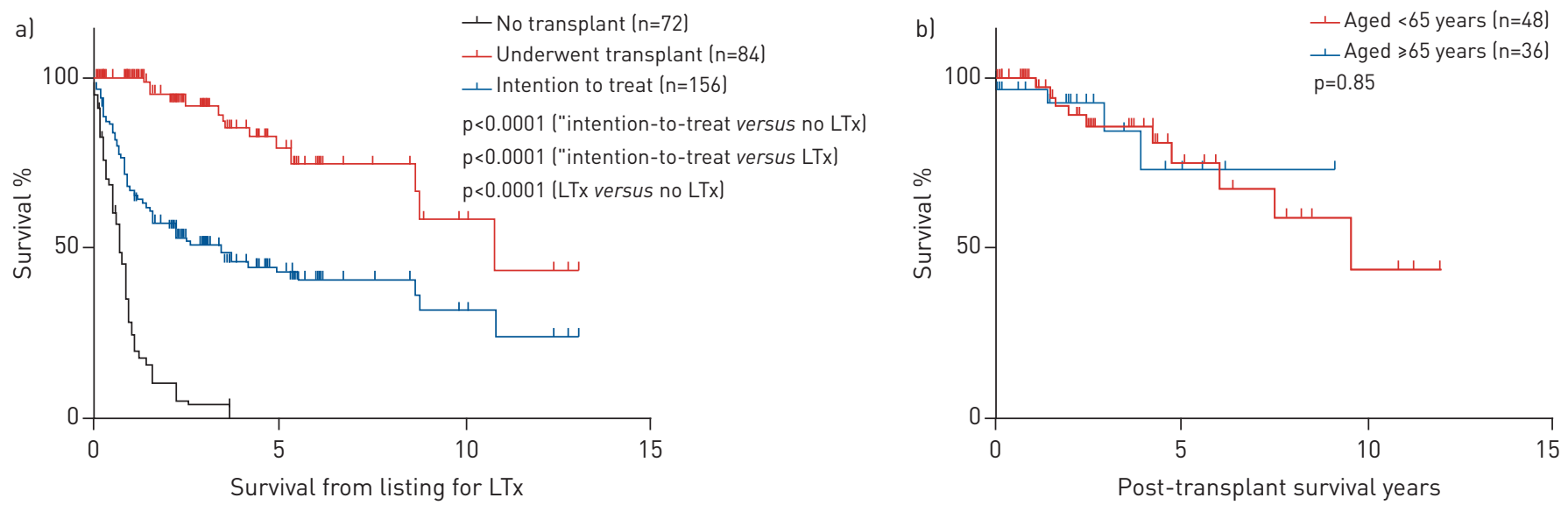

FIGURE 1 a) The survival curve of patients awaiting transplant, including time to death or transplant. b) Survival following single lung transplant ( $L T x$ ) for idiopathic pulmonary fibrosis, comparing patients aged $<65$ years versus those $\geqslant 65$ years at the time of transplant.

The 1-year survival of patients aged $<65$ years $(n=48)$ who underwent SLTx for IPF was $100 \%$, the 3-year survival was $85.4 \%$ (95\% CI $68.2-93.7 \%$ ) and the 5 -year survival was $75.1 \%$ (95\% CI 53.0-87.9\%). The 1 -year survival of patients aged $\geqslant 65$ years $(n=36)$ who underwent SLTx for IPF was $97.1 \%$ (95\% CI 80.9$99.6 \%$ ), the 3 -year survival was $84.4 \%$ (95\% CI 54.9-95.3\%) and the 5-year survival was $73.8 \%$ (95\% CI 39.6-90.6\%).There was no statistical difference in the post-transplant survival of IPF patients aged $<65$ years compared to those aged $\geqslant 65$ years $(\mathrm{p}=0.85)$ (figure $1 \mathrm{~b}$ ). The hazard ratio for death of IPF patients $\geqslant 65$ years compared to $<65$ years was 0.97 (95\% CI $0.31-3.06$ ).

This study highlights the survival benefit of SLTx for IPF patients. Without transplantation, the median survival from time of listing was 0.68 years. Over the course of this study $39.1 \%$ of IPF patients listed for transplant died on the waiting list. This contrasts with a Kaplan-Meier estimated median survival of 9.6 years following transplantation. When comparing outcomes of IPF patients transplanted at 65 years or older (range 65.1-75.7 years) to those under 65 (range 41.1-64.9 years), there was no difference in survival following transplant, rates of primary graft dysfunction, or acute/chronic rejection. The significant wait list mortality is commensurate with published experience [11]. There is no indication from the data characterising the disease burden that the patients $\geqslant 65$ years represent a cohort with an "immortality bias" (innate enhanced survival). Both groups of patients had matching death rates while awaiting transplant, comparable severity of impaired lung function, 6-min walk test and LAS. In the future we anticipate that the high mortality rate on the waiting list may be attenuated by the more widespread use of anti-fibrotic therapy. The intention-to-treat analysis emphasises the impact of waiting list mortality on outcome, highlighting the survival benefit that patients $\geqslant 65$ years can achieve with lung transplantation. Intention-to-treat analysis is informative, because demonstrating favourable outcomes following surgery underemphasises the importance of wait list mortality and skews outcome interpretation in favour of low risk patients

Our policy is that age should not preclude lung transplantation. However, particular emphasis is paid to rehabilitation status and physical functionality in individuals $\geqslant 65$ years of age. All patients adhered to a $\mathrm{BMI} \leqslant 28$, in order to optimise functional recovery post operatively. Immobile bed bound IPF patients would not be eligible for transplant. Patients should be assessed on an individual basis and transplantation offered to patients based on "biological age" rather than chronological age [12].

Donation rates and organ utilisation rate vary from country to country [13]. The deceased brain death donation rate in Ireland is 18 per million of population with a conversion rate of 38\% [13]. Donation rates may determine optimal organ utilisation and should support double lung transplantation in emphysema and/or cystic fibrosis patients. During this study, our strategy was to preferentially perform SLTx for IPF patients. This approach was focused on maximising donor utilisation and minimising surgical morbidity (associated with longer surgery) in an older aged group. During the study period, 60 patients with cystic fibrosis and 27 with emphysema underwent double lung transplant. While double lung transplant has been reported to increase post-transplant survival compared to SLTx for IPF patients, post-transplant outcomes in this study compare well to international data [14].

In summary, although our absolute sample size was small and additional limitations of our study include its single centre nature, potential selection bias and retrospective design, we were able to demonstrate a 
clinically meaningful survival benefit can be achieved with lung transplantation for IPF patients aged $\geqslant 65$ years.

Peter Riddell ๑, Jana Kleinerova, Donna Eaton, David G. Healy, Hossein Javadpour, Jim F. McCarthy, Lars Nolke, Karen C. Redmond and Jim J. Egan

National Heart and Lung Transplant centre, Mater Misericordiae University Hospital, Dublin, Ireland.

Correspondence: Jim J. Egan, National Heart and Lung Transplant centre, Mater Misericordiae University Hospital, Dublin, Ireland. E-mail: jegan@mater.ie

Received: 22 May 2018 | Accepted after revision: 26 Feb 2020

Conflict of interest: None declared.

\section{References}

1 King TE, Bradford WZ, Castro-Bernardini S, et al. A phase 3 trial of pirfenidone in patients with idiopathic pulmonary fibrosis. N Engl J Med 2014; 370: 2083-2092.

2 Richeldi L, du Bois RM, Raghu G, et al. Efficacy and safety of nintedanib in idiopathic pulmonary fibrosis. $N$ Engl J Med 2014; 370: 2071-2082.

3 Egan JJ, Martinez FJ, Wells AU, et al. Lung function estimates in idiopathic pulmonary fibrosis: the potential for a simple classification. Thorax 2005; 60: 270-273.

4 Thabut G, Mal H, Castier Y, et al. Survival benefit of lung transplantation for patients with idiopathic pulmonary fibrosis. J Thorac Cardiovasc Surg 2003; 126: 469-475.

5 Ley B, Collard HR, King TE. Clinical course and prediction of survival in idiopathic pulmonary fibrosis. Am J Respir Crit Care Med 2011; 183: 431-440.

6 Yusen $\mathrm{RD}$, Christie JD, Edwards LB, et al. The Registry of the International Society for Heart and Lung Transplantation: Thirtieth Adult Lung and Heart-Lung Transplant Report--2013; focus theme: age. J Heart Lung Transplant 2013; 32: 965-978.

7 Eurotransplant Statistics. Database Information Request; 12 March 2018. statistics.eurotransplant.org

8 Adamali HI, Judge EP, Healy D, et al. International collaboration: a retrospective study examining the survival of Irish citizens following lung transplantation in both the UK and Ireland. BMJ Open 2012; 2: e000605-e000605.

9 Organ Procurement and Transplantation Network. LAS Calculator. https://optn.transplant.hrsa.gov/resources/ allocation-calculators/

10 Gupta S. Intention-to-treat concept: a review. Perspect Clin Res 2011; 2: 109-112.

11 Mackay LS, Anderson RL, Parry G, et al. Pulmonary fibrosis: rate of disease progression as a trigger for referral for lung transplantation. Thorax 2007; 62: 1069-1073.

12 Wilson ME, Vakil AP, Kandel P, et al. Pretransplant frailty is associated with decreased survival after lung transplantation. J Hear Lung Transplant 2016; 35: 173-178.

13 Riddell P, Egan JJ. International donor conversion rates for lung transplantation need to be standardised. Lancet Respir Med 2015; 3: 909-911.

14 Chambers DC, Cherikh WS, Goldfarb SB, et al. The International Thoracic Organ Transplant Registry of the International Society for Heart and Lung Transplantation: Thirty-fifth adult lung and heart-lung transplant report -2018; focus theme: multiorgan transplantation. J Hear Lung Transplant 2018; 37: 1169-1183. 